\title{
MicroscopyPioneers \\ Pioneers in Optics: Charles Wheatstone
}

\author{
Michael W. Davidson
}

National High Magnetic Field Laboratory, Florida State University, Tallahassee, FL 32306

davidson@magnet.fsu.edu

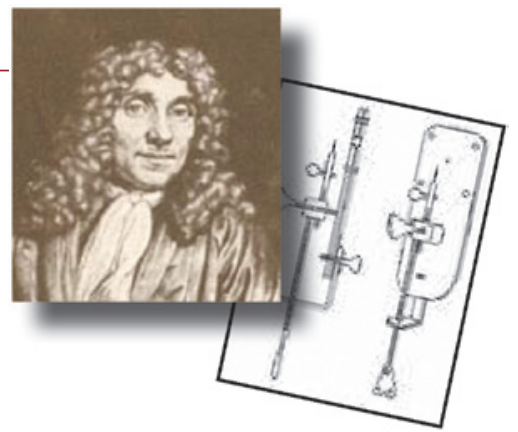

\section{Charles Wheatstone (1802-1875)}

Charles Wheatstone was a prominent nineteenthcentury physicist who made significant contributions to a number of areas without having ever received a formal scientific education. He was particularly influential in the field of optics, where he revolutionized contemporary notions of vision and spatial perception. Born February 6, 1802, in Gloucester, England, Wheatstone entered into an extended family that built and sold musical instruments. He was reared in the family trade, serving an apprenticeship with an uncle who manufactured string instruments. Extremely curious and inventive in his youth, Wheatstone

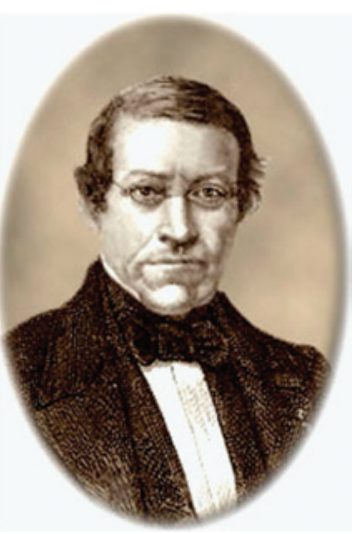
began his independent scientific development through avid reading and by carrying out experiments in acoustics.

In 1821, Wheatstone garnered a considerable amount of public attention by devising what was commonly referred to as the enchanted lyre. Part of an innovative acoustic show, the lyre was attached to the soundboards of a piano and other musical instruments in an upstairs room via a thin steel wire. When Wheatstone or another musician played music upstairs, the sound conduction and the sympathetic resonance of the strings made the lyre appear as if it were magically playing by itself. The feat was particularly important in the life of Wheatstone because it brought him into contact with renowned Danish scientist Christian Oersted who wanted to meet the man who devised it. When he found that his new acquaintance had carried out a number of experiments similar to his own, he encouraged Wheatstone to write a paper that reported his findings. The resulting work entitled New Experiments in Sound became Wheatstone's first scientific publication.

By 1834 Wheatstone had built such a reputation for himself that he was appointed professor of experimental physics at King's College in London. However, he proved to be a poor teacher, due to his innate shyness, and rarely lectured, although he retained the post for the rest of his life. Wheatstone's poor presentation ability also often prevented him from reading his own papers to academic societies. Instead, he frequently depended on his close friend and fellow scientist Michael Faraday to adequately portray his ideas.

No matter how lackluster his speaking skills, however, Wheatstone was a brilliant scientist. Intensely interested in electricity, in 1834 he made the first measurement of the velocity of an electrical current passing through a wire by using a rotating mirror. He later suggested that the same device be used to measure the speed of light. Wheatstone's other significant contributions in the field include improvements to the dynamo, the invention of an adjustable resistor known as the rheostat, and popularizing a method of measuring electrical resistance invented by Samuel Christie, which came to be known as the Wheatstone bridge.

Wheatstone was also highly concerned with optics, a field he originally entered because of his interest in expressing acoustic phenomena visually. His various studies and experiments led him to develop the theory of stereoscopic vision, which involves the idea that each eye sees a slightly different view of a single scene, which combine in a way that results in depth perception. He then used his new understanding of vision to invent the stereoscope, which he presented to the Royal Society in 1838 . The instrument was designed so that it could present a slightly different two-dimensional image to each eye, which the viewer would then interpret as a single three-dimensional picture. Primarily used for entertainment purposes, various versions of the stereoscope became extremely popular in Victorian England.

During the same period that he was developing his ideas regarding vision, Wheatstone was busily designing the first viable telegraph system in conjunction with William Cooke. The pair patented the five-needle telegraph and publicly demonstrated the device in 1837. Though theirs was the first operational commercial electric telegraph, it was later replaced by simpler technology, such as one invented by Samuel Morse. However, the partners made improvements on their system for many years to come. In fact, when Wheatstone died of bronchitis on October 19, 1875, he was in Paris on a business trip promoting his latest developments to French telegraph authorities. 


\section{MICROSCOPES \\ I N T E R N A T I O N A L}

\section{Isn't it about time you had your own Digital Microscope?}

Now you can with the portable, affordable uScopeMXII!

The uScopeMXII is a small digital desktop microscope you can use in your workplace or home office. It captures images from standard glass slides and sends them to your PC.

You can interactively browse slides with full control of focus, image processing, and location. You can also scan regions of interest creating fully focused image sets.

The industry-standard USB interface makes it simple to plug in and start capturing images. It easily interfaces with your desktop or laptop PC and allows you to view and capture slide images in a wide variety of environments.

The uScopeMXII is manufactured in the United States.

\section{Features and Benefits}

- Overview and Objective Cameras The uScopeMXII has an objective camera for scanning and an overview camera for navigating.

\section{- Automatic Focus}

Images are automatically focused using configurable focus algorithms.

\section{- Portability}

At a weight of about $5 \mathrm{lbs}$., the uScopeMXII is highly portable.

- Easy to Use The uScope Navigator software simplifies scanning and browsing.

- Self-Contained

The uScopeMXII is self-contained and includes the electronics, cameras, stage, and optics in a device about the size of a large external disk drive.

- Full Imaging Control

User filters provide complete control over image processing and correction.

\section{Microscopes International, LLC}

555 Republic Drive, Suite 119

Plano, TX 75074-5498

Phone +1-214-785-2058

FAX +1-214-785-2138

Emailssales@uscopes.com support@uscopes.com
(6)

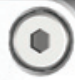

MICROSCOPES

$\mathrm{uSCOPE}^{\mathrm{m}}{ }_{\mathrm{m} \times 1}$

- POWER

- STATUS

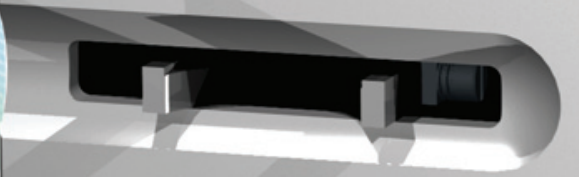

Call us today for a quote or to schedule a demonstration 